\title{
A Review of Cloud Deployment Models for E-Learning Systems
}

\author{
Engin Leloglu \\ Dept. of Computer Engineering \\ Izmir Institute of Technology \\ Izmir, TURKEY \\ enginleloglu@ieee.org
}

\author{
Tolga Ayav \\ Dept. of Computer Engineering \\ Izmir Institute of Technology \\ Izmir, TURKEY \\ tolgaayav@iyte.edu.tr
}

\author{
Burak Galip Aslan \\ Dept. of Computer Engineering \\ Izmir Institute of Technology \\ Izmir, TURKEY \\ bgaslan@ieee.org
}

\begin{abstract}
With the significant growth in the cloud-based systems, many industries give their attention to cloud computing solutions. E-learning is a promising application area since its typical requirements such as dynamically allocation of computation and storage resources, coincide well with cloud characteristics. This paper presents some possible cloud solutions in e-learning environments by emphasizing its pros and cons. It is of paramount importance to choose the most suitable cloud model for an e-learning application or an educational organization in terms of scalability, portability and security. We distinguish various deployment alternatives of cloud computing and discuss their benefits against typical e-learning requirements.
\end{abstract}

Keywords-component; cloud computing; e-learning; deployment models

\section{INTRODUCTION}

Recent progresses in Internet technology give an opportunity to access an environment that allows users to develop and run applications. This environment includes data centers that are monitored and maintained by content providers [1]. Cloud computing is a promising infrastructure which is based on this paradigm. Interactivity and collaboration are major points of this new technology [2].

E-learning, which is the new trend of the education, should keep up pace with the technology to satisfy the needs and improve the quality of learning. In this sense, e-learning systems requirements coincide with Cloud computing benefits.

There are several cloud computing service providers that offer support for educational systems such as Amazon, Google, Yahoo and Microsoft. They provide services to facilitate the development of scalable web applications in a dynamic and virtualized sense.

The following sections include cloud computing concepts and principles of cloud e-learning solutions. Also, alternative approaches for cloud-based e-learning systems have been discussed.

\section{Cloud Computing}

Cloud computing is a model for enabling convenient, ondemand network access to a shared pool of configurable computing resources (e.g., networks, servers, storage, applications, and services) that can be rapidly provisioned and released with minimal management effort or service provider interaction [3]. More recently, three cloud deployment models have been found admissible in the Cloud market:

1) Public cloud: This model is used by the general public cloud consumers and the cloud service provider has the full ownership of the public cloud with its own policy, value, and profit, costing, and charging model [4]. It is the most common model of Cloud computing.

2) Private cloud: The cloud infrastructure is operated solely within a single organization, and managed by the organization or a third party regardless whether it is located premise or off premise [4]. Especially security and privacy concepts enforce organizations to use this model of cloud computing.

3) Hybrid cloud: The cloud infrastructure is a combination of two or more clouds (private or public) that remain unique entities but are bound together by standardized or proprietary technology that enables data and application portability [4].

The customers can choose one of cloud deployment models, depending on their requirements.

\section{AN OVERVIEW OF CLOUd-BASED E-LEARNING SOLUTIONS}

Many education institutions do not have sufficient resources and desired infrastructure needed to run popular elearning solutions. This is why the biggest players in the field of e-learning software have now versions of the base applications that are cloud oriented.

Some important merits with respect to cloud-based elearning solutions are given below [5];

1) Lower costs: You don't need a high-powered and high-priced computer to run cloud computing web-based applications, since applications run in the cloud, not on the desktop PC

2) Improved performance: Cloud-based e-learning systems boot and run faster because they have fewer programs and processes loaded into device memory.

3) Instant software updates: When the app is web-based, updates occur automatically and are available the next time you $\log$ on to the cloud. 
4) Increased data reliability: Even, if the personal computer crashes, all data is still intact in the cloud, still accessible.

5) Device independence: You're no longer tethered to a single computer or network. Change computers, and your existing applications and documents follow you through the cloud.

6) Improved improbability: It is almost impossible for any unauthorized person to have access any data by finding out digital assets (tests, exam questions, results) [2].

However, there are some risks besides these benefits;

1) Network: Internet connections are required, and stable ones are often essential. Also, if a Cloud connection gets terminated during a session, users may lose time, work, or even unsaved data [6].

2) Security: Many organizations feel insecure with the idea of storing their data and applications on systems that they do not have full control [7].

3) Portability: The ability to bring systems back inhouse or choose another cloud provider will be limited by proprietary interfaces [7].

\section{Cloud-Based E-LeARning System Alternatives}

\section{A. Public Cloud Approach}

Choice of public cloud model is the most practical approach to get the quickest solution to develop a reasonable cloud-based e-learning system. When the requirements under product development are considered, this choice makes developers benefit from a Cloud solution in a quickest and lowest cost. However, this approach provides a reason of discomfort for organization owners. Migrating workloads to a shared infrastructure increases the potential for unauthorized access and exposure. It is interpreted as a risk of security or privacy. Also, once an organization builds or transfers a system to use the benefits of public cloud provider's offerings, bringing that system back in-house will be relatively difficult and expensive [7].

\section{B. Private Cloud Approach}

Operated Cloud within a single organization and controlled Cloud by the same organization or a third party provides nearly secure and portable cloud-based e-learning system [8]. On the other hand, relatively higher costs are an important drawback of this approach. Since the private cloud is hosted on sight, the organization needs to provide adequate power, cooling, and general maintenance. Hence, the host organization also runs the risk of data loss due to physical damage of the unit. This data may contain crucial digital assets such as tests, exam questions, results, etc. [9].

\section{Hybrid Cloud Approach}

This approach provides an ease for bringing the e-learning system back-in house or transferring to another cloud provider by decreasing platform dependence. Also, it helps even increasing the system security. Hence, hybrid cloud model provides an environment to build a national private cloud system and operating system. However, considering that hybrid model is a combination of public and private models, distribution of units between these models is significant to address the requirements of the organization. Also, governance and management the other issues, inasmuch as there are two different models in use. It means that more expertise and increased consultancy costs are needed to install and maintain the system.

\section{CONCLUSION}

Cloud computing has an essential scope to make a breakthrough the whole education system. Hence, e-learning solutions cannot decline the cloud computing trends. To verify this idea, cloud computing concepts are emphasized and evaluated in this paper with respect to principles and requirements of e-learning solutions. The comparison of deployment models, depending on e-learning requirements, is articulated exhaustively. However, debates about Cloud deployment models still have not come to an end. It intensifies on the trade-offs about the cost and the risk management.

Creating experimental environments and measuring the effectiveness of these systems will eventually lead to gathering practical information for taking next steps. Critiques of people who get involved in these experiments should be taken in consideration and the success of learners should be observed for different conditions. Hence, scientific approaches can be applied much more effectively to real-world solutions.

In this respect, cloud-based e-learning systems will help the students, teachers, institutions and learners who live in rural parts of the world reach the knowledge shared by experts, professors etc. Governments will eventually start installing and using such systems in schools and colleges in the near future.

\section{REFERENCES}

[1] Al-Zoube, M.: "E-Learning on the Cloud", International Arab Journal of e-Technology, Vol. 1, No. 2, pp. 58-64, June 2009.

[2] Pocatilu, P., Alecu, F., Vetrici, M.: "Measuring the Efficiency of Cloud Computing for E-learning Systems", WSEAS TRANSACTIONS on COMPUTERS, Issue 1, Volume 9, pp. 42-51, January 2010.

[3] Mell, P., Grance, T.: "The NIST Definition of Cloud Computing" National Institute of Standards and Technology Special Publication 800145, September 2011.

[4] Dillon, T., Wu, C., Chang, E.: "Cloud Computing: Issues and Challenges", 24th IEEE International Conference on Advanced Information Networking and Applications, pp. 27-33, 2010.

[5] Rao, N.M., Sasidhar, C., Kumar, V.S.: "Cloud Computing Through Mobile-Learning", (IJACSA) International Journal of Advanced Computer Science and Applications Vol.1, No. 6, pp. 42-47, December 2010.

[6] Campbell, S.: "e-Learning in "the Cloud", http://www.syberworks.com/articles/elearning-in-the-cloud-article.htm, September 2012, unpublished.

[7] Open Cloud Manifesto, http://www.opencloudmanifesto.org, September 2012, unpublished.

[8] Şanl1, O.: “A view over the Clouds", Attended Academic IT Conference, February, 2012.

[9] Lupacchino, M.: "Cloud Computing 101: Private Vs. Public", http://blog.nskinc.com/IT-Services-Boston/bid/26417/CloudComputing-101-Private-Vs-Public, March 2010, unpublished. 